\title{
Higher cotangent cohomology of rational surface singularities
}

\author{
Jan Stevens
}

\begin{abstract}
We give dimension formulas for the cotangent cohomology groups $T^{i}$ for all rational surface singularities. The computations take place on the first blow-up of the singularity.
\end{abstract}

\section{Introduction}

The modules $T^{1}$ and $T^{2}$ play an important role in deformation theory, the first as a space of infinitesimal deformations, while the obstructions land in the second. Much work has been done to compute their dimension for rational surface singularities, culminating in the formulas of Christophersen and Gustavsen [CG01]. The 'correct' way to define $T^{1}$ and $T^{2}$ also yields higher $T^{i}$. The purpose of this paper is to generalize the dimension formulas to these modules.

For rational surfaces with reduced fundamental cycle, De Jong and Van Straten [JS94] showed that the dimension of $T^{2}$ can be computed inductively from the multiplicities of the singularities on the successive blow-ups. In the general case of a rational surface singularity $X$ of multiplicity $d \geqslant 3$ with first blow-up $\widehat{X}$ the result [CG01, Theorem 3.8] is

$$
\operatorname{dim} T_{X}^{2}=(d-1)(d-3)+\operatorname{dim} T_{\widehat{X}}^{2}+c(X),
$$

where $c(X)$ is an unavoidable correction term, which vanishes for large classes of singularities (including reduced fundamental cycle). Here $T_{\widehat{X}}^{2}$ is the direct sum of the local $T_{\widehat{X}, x}^{2}$ at the (isolated) singular points of $\widehat{X}$. With the same correction term one has [CG01, Theorem 3.13]:

$$
\operatorname{dim} T_{X}^{1}=(d-3)+\operatorname{dim} T_{\widehat{X}}^{1}+c(X) .
$$

In this case the global $T_{\widehat{X}}^{1}$ can be computed from a local-to-global sequence, which involves a non-zero global contribution. The group $T_{\widehat{X}}^{1}$ is the Zariski tangent space of the base of the versal deformation of the space $\widehat{X}$.

Our main result is that a corresponding formula holds for the higher $T^{i}$ without correction term:

$$
\operatorname{dim} T_{X}^{i}=f_{i}(d)+\operatorname{dim} T_{\widehat{X}}^{i}
$$

with $f_{i}(d)$ an explicit function of $d$. If $\widehat{X}$ is smooth, then $\operatorname{dim} T_{X}^{i}$ equals $f_{i}(d)$, so the value of $f_{i}(d)$ follows from the computations in [AS99] for the cone over the rational normal curve of degree $d$; see also $\S 1.6$.

We follow the arguments of [CG01] closely, replacing the computations of the different $T^{2}$ 's in terms of functions on relations by computations with Harrison cohomology. By means of a Noether normalization (a flat map $X \rightarrow S$ of degree $d$ onto a smooth surface) it suffices to look at relative Harrison cohomology, whose defining complex is $\mathcal{O}_{S}$-linear and therefore much smaller. We use here

Received 24 June 2002, accepted in final form 24 December 2002.

2000 Mathematics Subject Classification 32S30, 32S25.

Keywords: Harrison cohomology, cotangent complex, rational singularity.

The author was partially supported by the Swedish Research Council (Vetenskapsrådet).

This journal is (c) Foundation Compositio Mathematica 2004. 


\section{Higher COTANGENT COHOMOLOGY}

in an essential way the fact that the iterated hyperplane section of a rational surface singularity is the fat point of minimal multiplicity $d$. The computations with relative Harrison cohomology work well for $i \geqslant 2$. Our arguments reprove the $T^{2}$-formula from [CG01].

De Jong and Van Straten [JS94] prove their formulas for $T^{1}$ and $T^{2}$ with a one-parameter deformation of the singularity (with reduced fundamental cycle) to the cone over the rational normal curve of degree $d$ and all singularities on the first blow-up. In this deformation the codimension of the Artin component and the dimension of $T^{2}$ are constant. By induction on the singularities on the blow-up it then follows that the number of equations needed for the base space equals the dimension of $T^{2}$, a property known as the surjectivity of the obstruction map. Surjectivity still follows if we forget about rational double points and relax the requirements. It was hoped that every rational surface singularity admits such a good maximal deformation, but examples show that this is not the case.

\section{Cotangent cohomology}

1.1 The definition of $T_{X}^{i}$ is most conveniently given in the context of a more general theory and we therefore set $T_{X}^{i}:=T^{i}\left(X / \mathrm{pt} ; \mathcal{O}_{X}\right)$. Let $X \rightarrow S$ be a map of analytic germs and $M$ an $\mathcal{O}_{X}$-module. One gets the cotangent cohomology groups as

$$
T^{i}(X / S ; M):=H^{i}\left(\operatorname{Hom}_{\mathcal{O}_{X}}\left(\mathbb{L}_{*}^{X / S}, M\right)\right)
$$

with $\mathbb{L}_{*}^{X / S}$ being the so-called cotangent complex. All we need to know about is summarised in [BC91, CG01]. A good reference for this section and the next is [Lod92]. We actually work with the analytic version of the cotangent complex, which can be constructed from a Tyurina resolvent of the analytic $\mathcal{O}_{S}$-algebra $\mathcal{O}_{X}$, see Palamodov's survey [Pal90] and for more details his papers [Pal76, Pal83, Pal84].

1.2 The higher cotangent cohomology can also be computed as Harrison cohomology. To give the definition, we first recall Hochschild cohomology. Let $A$ be a commutative algebra of essentially finite type over a base ring $k$. For an $A$-module $M$, the Hochschild cohomology $H H^{i}(A / k ; M)$ is the cohomology of the complex

$$
C^{i}(A / k ; M):=\operatorname{Hom}_{k}\left(A^{\otimes i}, M\right)
$$

with differential

$$
(\delta f)\left(a_{0}, \ldots, a_{i}\right)=a_{0} f\left(a_{1}, \ldots, a_{i}\right)+\sum_{j=1}^{i}(-1)^{j} f\left(a_{0}, \ldots, a_{j-1} a_{j}, \ldots, a_{i}\right)+(-1)^{i+1} a_{i} f\left(a_{0}, \ldots, a_{i-1}\right) .
$$

The same cohomology can also be obtained from the so-called reduced subcomplex $\bar{C}^{\bullet}(A / k ; M)$ consisting only of those maps $f: A^{\otimes i} \rightarrow M$ that vanish whenever at least one of the arguments equals 1 .

Definition 1.1. A permutation $\sigma$ is called a $(p, q)$-shuffle if $\sigma(1)<\cdots<\sigma(p)$ and $\sigma(p+1)<\cdots<$ $\sigma(p+q)$. Moreover, in the group algebra $\mathbb{Z}\left[S_{p+q}\right]$ we define the elements

$$
\operatorname{sh}_{p, q}:=\sum_{(p, q) \text {-shuffles }} \operatorname{sgn}(\sigma) \sigma .
$$

These elements give rise to the so-called shuffle invariant subcomplexes

$$
C_{\mathrm{sh}}^{i}(A / k ; M):\left\{f \in \operatorname{Hom}_{k}\left(A^{\otimes i}, M\right) \mid f \circ \operatorname{sh}_{p, i-p}=0 \text { for } 0<p<i\right\}
$$

and $\bar{C}_{\mathrm{sh}}^{i}(A / k ; M) \subset \bar{C}^{i}(A / k ; M)$ defined in the same manner. Both complexes yield the same cohomology, which is called Harrison cohomology $\operatorname{Harr}^{i}(A / k ; M)$. 


\section{J. Stevens}

Proposition 1.2. If $\mathbb{Q} \subset k$, then Harrison cohomology is a direct summand of Hochschild cohomology.

In the analytic case one has to use the analytic tensor product; precise definitions are given by Palamodov [Pal83]. We can now state the relation with cotangent cohomology.

Theorem 1.3. If the map $X \rightarrow S$ is flat, then

$$
T^{i}(X / S ; M) \cong \operatorname{Harr}^{i+1}\left(\mathcal{O}_{X} / \mathcal{O}_{S} ; M\right) .
$$

1.3 Let $X$ be a Cohen-Macaulay singularity of dimension $n$ and multiplicity $d$. We can choose as Noether normalization $X \rightarrow \mathbb{C}^{n}$ a flat map of degree $d$. In fact, this is only a map of germs of analytic spaces, but we are abusing notation here. The exact sequence relating absolute and relative cotangent cohomologies gives, for an $\mathcal{O}_{X}$-module $M$,

$$
\cdots \rightarrow T^{i-1}\left(\mathbb{C}^{n} ; M\right) \rightarrow T^{i}\left(X / \mathbb{C}^{n} ; M\right) \rightarrow T^{i}(X ; M) \rightarrow T^{i}\left(\mathbb{C}^{n} ; M\right) \rightarrow \cdots .
$$

As $\mathbb{C}^{n}$ is smooth, $T^{i}\left(\mathbb{C}^{n} ; M\right)=0$ for $i \geqslant 1$. We therefore obtain the following.

Lemma 1.4. One has $T^{i}(X ; M) \cong T^{i}\left(X / \mathbb{C}^{n} ; M\right)$ for $i \geqslant 2$ for any $\mathcal{O}_{X}$-module $M$.

In particular, if $X$ has minimal multiplicity $d$ (e.g., a rational surface singularity), so embdim $X=$ $d+n-1$, we may choose coordinates $\left(z_{1}, \ldots, z_{d+n-1}\right)$ such that the projection on the space spanned by the last $n$ coordinates is a Noether normalization.

In terms of rings we have a regular local ring $P=\mathbb{C}\left\{z_{d}, \ldots, z_{d+n-1}\right\}$ and a homomorphism $P \rightarrow A$ making the local ring $A$ of $X$ into a free $P$-module of rank $d$, with basis $\left\{1, z_{1}, \ldots, z_{d-1}\right\}$. The equations for $X$ are of the form

$$
z_{i} z_{j}=\sum_{\nu=1}^{d-1} p_{\nu} z_{\nu}+p_{0}, \quad 1 \leqslant i, j \leqslant d-1,
$$

where $p_{\nu} \in P$ for $\nu=0, \ldots, d-1$.

1.4 Let $X$ be a rational surface singularity of multiplicity $d \geqslant 3$ with local $\operatorname{ring} A$ and Noether normalization $X \rightarrow \mathbb{C}^{2}$, or in terms of rings $P \rightarrow A$. We can obtain the higher cotangent cohomology as Harrison cohomology $\operatorname{Harr}^{i+1}(A / P ; M)$ computed from the reduced complex. As an illustration of this technique we repeat here the proof of the following lemma, shown in [AS99]. We shall need the argument later on.

Lemma 1.5. The natural map $\operatorname{Harr}^{i+1}(A / P ; A) \rightarrow \operatorname{Harr}^{i+1}(A / P ; \mathbb{C})$ is the zero map.

Proof. As $\left\{1, z_{1}, \ldots, z_{d-1}\right\}$ forms a basis of $A$ as $P$-module, a reduced Harrison $(i+1)$-cocycle $f$ is, by $P$-linearity, determined by its values on the $(i+1)$-tuples of the coordinates $z_{1}, \ldots, z_{d-1}$. Suppose $f\left(z_{j_{0}}, \ldots, z_{j_{i}}\right) \notin \mathfrak{m}_{A}$. Since $d \geqslant 3$, we may choose a $z_{k}$ with $k \in\{1, \ldots, d-1\}$ and $k \neq j_{0}$. Hence,

$$
\begin{aligned}
0=(\delta f)\left(z_{j_{0}}, \ldots, z_{j_{i}}, z_{k}\right)= & z_{j_{0}} f\left(z_{j_{1}}, \ldots, z_{k}\right) \pm f\left(z_{j_{0}}, \ldots, z_{j_{i}}\right) z_{k} \\
& + \text { terms containing products } z_{i} z_{j} \text { as arguments. }
\end{aligned}
$$

Using the equations for $X$ as in $\S 1.3$ we may again apply $P$-linearity to see that the latter terms are contained in $\mathfrak{m}_{P} \cdot A$. Hence, modulo $\mathfrak{m}_{P}$, these terms vanish, but the resulting equation inside $V=\mathfrak{m}_{A} / \mathfrak{m}_{P} A$ contradicts the fact that $z_{j_{0}}$ and $z_{k}$ are linearly independent.

1.5 Another powerful tool is the comparison between the cotangent cohomology of a singularity and its hyperplane section [BC91]. Let $f: X \rightarrow \mathbb{C}$ be a flat map such that both $X$ and the special fibre $H$ have isolated singularities. 


\section{Higher COTANGENT COHOMOLOGY}

Main Lemma [BC91, $\S 1.3 .2]$. There is a long exact sequence

$$
T^{1}\left(X / \mathbb{C} ; \mathcal{O}_{X}\right) \rightarrow T_{H}^{1} \rightarrow T_{X}^{2} \stackrel{f}{\rightarrow} T_{X}^{2} \rightarrow T_{H}^{2} \rightarrow T_{X}^{3} \stackrel{f}{\rightarrow} T_{X}^{3} \rightarrow \cdots .
$$

Moreover, $\operatorname{dim} T_{X}^{2} /\left(f \cdot T_{X}^{2}\right)=\tau_{H}-e_{f}$ with $\tau_{H}:=\operatorname{dim} T_{H}^{1}$ and $e_{f}$ the dimension of the smoothing on which $f$ lies in the versal base space of $H$.

1.6 The Main Lemma becomes particularly useful if the maximal ideal of $X$ annihilates the $T_{X}^{i}$ for $i \geqslant 2$. In [AS99] it is shown that the cone over the rational normal curve of degree $d$ has this property. Moreover, a formula is given for the dimension of all $T^{i}$.

To formulate it we first consider the fat point $Z_{m}$ of minimal multiplicity $d=m+1$, which is the iterated hyperplane section of the cone over the rational normal curve. We define the number $c_{m, k}$ as the dimension of $T^{k-1}\left(Z_{m} ; \mathbb{C}\right)$. The dimension of $T_{Z_{m}}^{i}$ is then $m c_{m, i+1}-c_{m, i}$. By, e.g., [AS99, $\S 2.4]$

$$
c_{m, k}=\frac{1}{k} \sum_{q \mid k}(-1)^{k+k / q} \mu(q) m^{k / q} .
$$

The first few values are $c_{m, 1}=m, c_{m, 2}=\left(m^{2}+m\right) / 2, c_{m, 3}=\left(m^{3}-m\right) / 3, c_{m, 4}=\left(m^{4}-m^{2}\right) / 4$, $c_{m, 5}=\left(m^{5}-m\right) / 5$ and $c_{m, 6}=\left(m^{6}+m^{3}-m^{2}-m\right) / 6$. We combine them in the power series

$$
Q_{d}(t)=\sum_{i \geqslant 1} c_{d-1, i} t^{i}
$$

Proposition 1.7 [AS99, § 4.7]. The Poincaré series

$$
P_{d}(t)=\sum_{i \geqslant 1} f_{i}(d) \cdot t^{i}:=\sum_{i \geqslant 1} \operatorname{dim} T_{X_{d}}^{i} \cdot t^{i}
$$

of the cone $X_{d}$ over the rational normal curve is given by

$$
P_{d}(t)=\left(Q_{d}(t)+2 t+2\right) \frac{(d-1) t-t^{2}}{(t+1)^{2}}-\frac{2 t}{t+1} .
$$

For low values of $i$ we get as dimensions of $T_{X_{d}}^{i}$

$$
\begin{aligned}
& f_{1}(d)=2 d-4 \\
& f_{2}(d)=(d-1)(d-3) \\
& f_{3}(d)=(d-1)(d-2)(d-3) / 2 \\
& f_{4}(d)=(d-1)(d-2)\left(2 d^{2}-8 d+9\right) / 6 \\
& f_{5}(d)=(d-1)(d-2)^{2}\left(3 d^{2}-8 d+9\right) / 12 \\
& f_{6}(d)=(d-1)(d-2)\left(12 d^{4}-66 d^{3}+153 d^{2}-179 d+90\right) / 60 .
\end{aligned}
$$

For the cone $X_{d}$ the dimension of $T^{i}$ equals the number of generators of $T^{i}$ as $\mathcal{O}_{X}$-module. For an $\mathcal{O}_{X}$-module $M$ we denote the minimal number of generators, which is $\operatorname{dim} M / \mathfrak{m} M$, by $\operatorname{cg} M$. Feeding the above results into the Main Lemma and using the arguments of [BC91, $\S 5.1]$, one obtains the following (see [AS99]).

Theorem 1.8. For all rational surface singularities of multiplicity $d$ one has that $\operatorname{cg} T^{i}=f_{i}(d)$ for $i \geqslant 2$.

Furthermore, it is shown in [AS99, Theorem 5.3] that the dimension of $T^{i}, i \geqslant 3$, equals the number of generators for all rational surface singularities with the property that the tangent cone has only hypersurface singularities. 


\section{J. Stevens}

\section{The tangent complex with values in a sheaf}

2.1 To describe the relation between cotangent cohomology of a singularity and its first blow-up one has to globalize local constructions. We use the analytic cotangent complex of Palamodov; for a general overview see [Pal90], while some technical details are to be found in [Pal84].

Let $f: X \rightarrow S$ be a map of complex spaces. An analytic sheaf over $X$ is a morphism of complex

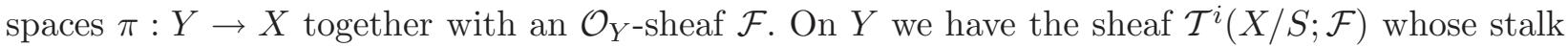
at a point $y \in Y$ is the cotangent cohomology $T^{i}\left(\mathcal{O}_{X, \pi(y)} / \mathcal{O}_{S, f \pi(y)} ; \mathcal{F}_{y}\right)$. This sheaf occurs in a local-to-global spectral sequence

$$
E_{2}^{p q}=R^{p}(f \pi)_{*}\left(\mathcal{T}^{q}(X / S ; \mathcal{F})\right) \Longrightarrow T^{i}(X / S ; \mathcal{F}) .
$$

In our application, where we will calculate the left-hand side, the right-hand side also reduces to a familiar object. Under the condition that $R^{q} f_{*}(\mathcal{F})=0$ for $q>0$, Christophersen and Gustavsen [CG01] obtain from Proposition 56 of the Appendix in [And74] that $T^{i}(X / S ; \mathcal{F}) \simeq$ $T^{i}\left(X / S ; f_{*} \mathcal{F}\right)$, where $f_{*} \mathcal{F}$ is a sheaf on $X$. For lack of reference we give here a proof using Palamodov's theory in the case that $f: X \rightarrow S$ is a finite map of germs.

2.2 A polyhedron $P$ in a complex space $X$ is a relatively compact subset $P \subset U \subset X$ together with a proper embedding $\varphi: U \rightarrow V \subset \mathbb{C}^{N}$, where $V$ is an open neighbourhood of the unit polydisc $D_{N}$, such that $P=\varphi^{-1}\left(D_{N}\right)$. A polyhedral covering $\mathcal{P}=\left\{P_{\alpha}, \alpha \in \mathcal{A}\right\}$ should satisfy $\bar{P}_{\beta} \subset U_{\alpha}$, if $\bar{P}_{\beta} \cap \bar{P}_{\alpha} \neq \emptyset$. A simplex of the nerve $\mathcal{N}(\mathcal{P})$ is a map $A:[n] \rightarrow \mathcal{A}$, written $A=\left(\alpha_{0}, \ldots, \alpha_{n}\right)$, such that $\bigcap \bar{P}_{\alpha_{i}} \neq \emptyset$. The set $P_{A}=\bigcap P_{\alpha_{i}}$ is again a polyhedron with $\varphi_{A}=\prod \varphi_{\alpha_{i}}: \bigcap U_{\alpha_{i}} \rightarrow \prod V_{\alpha_{i}} \subset \mathbb{C}^{N_{A}}$, where $N_{A} \sum N_{\alpha_{i}}$.

Given a map $f: X \rightarrow S$ and a polyhedron $Q \subset S$, a relative polyhedron over $Q$ is a subset $P$ with $\varphi: U \rightarrow V \supset D_{N}$ such that $\varphi \times f: U \rightarrow V \times S$ is a proper embedding and $P=\varphi^{-1}\left(D_{N}\right) \cap f^{-1}(Q)$. A polyhedral covering $\mathcal{P}$ of $X / S$ over a covering $\mathcal{Q}$ of $S$ is a covering by relative polyhedra such that each $P_{\alpha}$ lies over some $Q_{\nu(\alpha)}$. The mapping $\nu$ between index sets induces a morphism $\nu: \mathcal{N}(\mathcal{P}) \rightarrow$ $\mathcal{N}(\mathcal{Q})$.

A resolving sheaf for $X / S$ is a functor $\mathcal{R}$ defined on $\mathcal{N}(\mathcal{P})$ with values in the category of sheaves of graded differential algebras, such that each $\mathcal{R}_{A}$ is a free graded commutative algebra with a distinguished system of generators $e(A)$ of negative grading with differential $s$ such that the complex $\left(\mathcal{R}_{A}, s\right)$ is a resolution of the sheaf

$$
\mathcal{O}_{A}:=\left.\left(\varphi_{A} \times f\right)_{*} \mathcal{O}(X)\right|_{\bar{D}_{A} \times \bar{Q}_{\nu(A)}} .
$$

Furthermore, for $B \subset A$ each generator of $\mathcal{R}_{B}$ is mapped to a generator of $\mathcal{R}_{A}$ under the morphism $\left(p_{B}^{A}\right)^{*} \mathcal{R}_{B} \rightarrow \mathcal{R}_{A}$, where $p_{B}^{A}: \mathbb{C}^{N_{A}} \rightarrow \mathbb{C}^{N_{B}}$ is the canonical projection, and the map $e(B) \rightarrow e(A)$ is injective. The elements in $e(A)$ which are not in the image of any of the maps $e(B) \rightarrow e(A)$, with $B$ a proper subsimplex, are called proper generators. The functor $\mathcal{R}$ is completely determined by giving all the proper generators $e_{A, j}$ and the values $s\left(e_{A, j}\right)$. The proof of the existence of a resolving sheaf for $X / S$ for any polyhedral covering $\mathcal{P}$ of $X$ over a covering $\mathcal{Q}$ of $Y$ in [Pal84, Theorem 1.1] follows closely the absolute case in [Pal76].

Let $\rho_{A}: D_{A} \times Q_{\nu(A)} \rightarrow Y$. The functor $R$ from $\mathcal{N}(\mathcal{P})$ into the category of graded $\mathcal{O}_{Y}$ algebras, whose value on $A$ is $R_{A}:=\left(\rho_{A}\right)_{*}\left(\left.\mathcal{R}_{A}\right|_{\left.D_{A} \times Q_{\nu(A)}\right)}\right)$, is called the resolution of $X / S$ on $\mathcal{P}$.

Now let $Y \stackrel{\pi}{\rightarrow} X \stackrel{f}{\rightarrow} S$ and $\mathcal{F}$ be a sheaf on $Y$. Let $R$ be a resolution of $X / S$ on some polyhedral covering $\mathcal{P}, \mathcal{M}$ a polyhedral covering of $\pi$ over $\mathcal{P}$ and $\kappa: \mathcal{N}(\mathcal{M}) \rightarrow \mathcal{N}(\mathcal{P})$ the induced map between the nerves of the coverings. The functor $F=\pi_{*} \mathcal{F}(\mathcal{M})$ has value $\pi_{*} \mathcal{F}\left(M_{A}\right)$ on $A \in \mathcal{N}(\mathcal{M})$. Finally, $f_{*} F$ is a functor from $\mathcal{N}(\mathcal{M})$ to the category of $\mathcal{O}_{S}$-sheaves. 


\section{Higher Cotangent COHOMOLOGY}

Definition 2.1 [Pal84, Definition 2.2]. The tangent complex $T^{*}(R, F)=\sum_{0}^{\infty} T^{n}(R, F)$ of the resolution $R$ with values in $\mathcal{F}$ has as term of degree $n$ the $\mathcal{O}_{S}$-sheaf of $\mathcal{O}_{S}$-derivations of functors $\kappa^{*} R \rightarrow f_{*} F$ of degree $n$. In other words, an element $v \in T^{n}(R, F)_{s}$ is a collection of compatible $\mathcal{O}_{S, s}$-derivations $v_{A}: R_{\kappa(A), s} \rightarrow(f \pi)_{*}\left(\mathcal{F} \mid \mathcal{M}_{A}\right)_{s}$ for $A \in \mathcal{N}(\mathcal{M})$. The differential in the tangent complex is given by $d v=-(-1)^{\operatorname{deg} v} v s$ with $s$ the differential in $R$. The cohomology of this complex is $T^{n}(X / S ; \mathcal{F})$.

2.3 Now let $f:(X, x) \rightarrow(S, s)$ be a finite map of germs. We can choose the coverings $\mathcal{P}$ of $X$ and $\mathcal{Q}$ of $S$ to consist of one element each.

Proposition 2.2. There exists a spectral sequence

$$
E_{2}^{p, q}=T^{p}\left(X / S ; R^{q} \pi_{*} \mathcal{F}\right) \Longrightarrow T^{n}(X / S ; \mathcal{F})
$$

Proof. To compute the tangent complex $T^{*}(R, F)$ we describe the resolving sheaf $\mathcal{R}$ in more detail. Let $P_{a}$ be the polyhedron covering $X$. Let $\mathcal{R}_{a}$ be a resolution of $\mathcal{O}_{a}$ with generators $e_{a, j}$. This is basically the Tyurina resolvent of $\mathcal{O}_{X, x}$. The only simplices occurring in $\mathcal{N}(\mathcal{P})$ are of the form $A=(a, \ldots, a)$. The proper generators of $\mathcal{R}_{A}$ are divided into a basic group, consisting of $e_{A, j}$ with $\operatorname{deg} e_{A, j}=\operatorname{deg} e_{a, j}-\operatorname{dim} A$, and a complementary group of elements of degree $-\operatorname{dim} A$, corresponding to the coordinate functions $z_{j}$ on $\mathbb{C}^{n_{a}}$. By considering the $z_{j}$ as complementary generators of $\mathcal{R}_{a}$ of degree zero we have the relation $\operatorname{deg} e_{A, j}=\operatorname{deg} e_{a, j}-\operatorname{dim} A$ for all proper generators. The improper generators of $\mathcal{R}_{A}$ are of the form $e_{B, j}$ with $B \subset A$ a proper subsimplex.

We introduce a filtration $\psi$ on $e(A)$ by setting $\psi\left(e_{B, j}\right)=\operatorname{deg} e_{B, j}+\operatorname{dim} B$, which is the degree of the corresponding generator of $\mathcal{R}_{a}$. This is the same filtration as in the proof of [Pal84, Theorem 1.1], but this special case is simpler because there are no elements with filtration 1 . For all $k \leqslant 0$, we denote by $\mathcal{S}_{A}^{k}$ the subalgebra of $\mathcal{R}_{A}$ generated by the identity and the generators of filtration at least $k$. We define a derivation $\partial_{A}$ by

$$
\partial_{A}\left(e_{B, j}\right)=(-1)^{m} e_{B \backslash \beta_{0}, j}+(-1)^{m-1} e_{B \backslash \beta_{1}, j}+\cdots+e_{B \backslash \beta_{m}, j},
$$

where $B=\left(\beta_{0}, \ldots, \beta_{m}\right)$. The proof of [Pal84, Theorem 1.1] yields the fact that the differential in $\mathcal{R}_{A}$ can be written as $s_{A}=\partial_{A}+g_{A}$ with $g_{A}$ a derivation such that $g_{A}\left(e_{A, j}\right)$ is a section of $\mathcal{S}_{A}^{k+1}$ if $\psi\left(e_{A, j}\right)=k$.

Note that our $\partial_{A}$ differs from that in [Pal84], in that it extends to the last index. We indicate the first few terms of the resolution. Let $A=(a, a)$. To distinguish between the indices we write $A=(\alpha, \beta)$. On $\mathbb{C}^{N_{a}}$ we have coordinates $z_{j}$. The map $\varphi_{A}$ embeds $U_{A}=U_{a}$ in the diagonal of $\mathbb{C}^{N_{a}} \times \mathbb{C}^{N_{a}}$, so if we take coordinates $z_{j}^{(\alpha)}$ and $z_{j}^{(\beta)}$ we get the $N_{a}$ equations $z_{j}^{(\alpha)}-z_{j}^{(\beta)}$, which equals $\partial_{A}\left(z_{j}^{(A)}\right)$. To each generator $f$ of the ideal of $X$ there corresponds a generator $e_{A, f}$. One has that $s_{A}\left(e_{\alpha, f}\right)=f\left(z^{(\alpha)}\right)$ and $s_{A}\left(e_{\beta, f}\right)=f\left(z^{(\beta)}\right)$, and that $f\left(z^{(\beta)}\right)-f\left(z^{(\alpha)}\right)$ lies in the ideal of the diagonal, so it equals $\sum_{j}\left(z_{j}^{(\alpha)}-z_{j}^{(\beta)}\right) h_{j}^{(A)}$ for some functions $h_{j}^{(A)}$. Therefore, $s_{A}\left(e_{A, f}\right)=$ $-e_{\beta, f}+e_{\alpha, f}+\sum_{j} h_{j}^{(A)} z_{j}^{(A)}$.

The filtration $\psi$ induces a decreasing filtration on $T^{*}(R, F)$, of derivations $\kappa^{*} R \rightarrow f_{*} F$ vanishing on the functor $\kappa^{*} S^{-k}$. In its spectral sequence the differential $d_{0}$ is the differential of the Cech complex of the covering $\mathcal{M}$ of $Y$. The $E_{2}$ term is therefore $T^{p}\left(X / S ; R^{q} \pi_{*} \mathcal{F}\right)$.

\section{The formula}

3.1 We apply the results of the preceding section in the case that $X$ is a rational surface singularity of multiplicity $d$ and $\pi: \widehat{X} \rightarrow X$ is the first blow-up. Then $\pi_{*} \mathcal{O}_{\widehat{X}}=\mathcal{O}_{X}$ and $R^{q} \pi_{*}\left(\mathcal{O}_{\widehat{X}}\right)=0$ for $q>0$. We choose a Noether normalization $X \rightarrow S \cong \mathbb{C}^{2}$. Note that the induced map $\widehat{X} \rightarrow \widehat{S}$ to 


\section{J. Stevens}

the blow-up of the plane is again a finite map of degree $d$, so we can compute the higher cotangent cohomology sheaves of $\widehat{X}$ also as relative Harrison cohomology: $\mathcal{T}_{\widehat{X}}^{i} \cong \mathcal{T}^{i}\left(\widehat{X} / \widehat{S} ; \mathcal{O}_{\widehat{X}}\right)$ for $i \geqslant 2$.

By Proposition 2.2 we obtain $T^{i}\left(X / S ; \mathcal{O}_{\widehat{X}}\right)=T^{i}\left(X / S ; \mathcal{O}_{X}\right)$, which equals $T_{X}^{i}$ if $i \geqslant 2$. The local-to-global spectral sequence becomes

$$
E_{2}^{p, q}=H^{p}\left(\widehat{X}, \mathcal{T}^{q}\left(X / S ; \mathcal{O}_{\widehat{X}}\right)\right) \Longrightarrow T^{n}\left(X / S ; \mathcal{O}_{X}\right)
$$

The sheaves on $\widehat{X}$ occurring here are the basic objects of study. To shorten notation we write

$$
\mathcal{F}^{i}=\mathcal{T}^{i}\left(X / S ; \mathcal{O}_{\widehat{X}}\right)
$$

As only $H^{0}$ and $H^{1}$ contribute to the spectral sequence, we obtain the following (cf. [CG01, Corollary 1.7]).

Proposition 3.1. If $\pi: \widehat{X} \rightarrow X$ is the first blow-up of a rational surface singularity then one has short exact sequences

$$
0 \rightarrow H^{1}\left(\widehat{X}, \mathcal{F}^{i-1}\right) \rightarrow T^{i}\left(X / S ; \mathcal{O}_{X}\right) \rightarrow H^{0}\left(\widehat{X}, \mathcal{F}^{i}\right) \rightarrow 0
$$

for all $i \geqslant 0$.

Remark. Christophersen and Gustavsen [CG01] consider the absolute case. For $i \geqslant 2$ one has that $\mathcal{F}^{i} \cong \mathcal{T}^{i}\left(X ; \mathcal{O}_{\widehat{X}}\right)$. Comparison of the exact sequences for $T_{X}^{2}$ and $T^{2}\left(X / S ; \mathcal{O}_{X}\right)$ shows that even $H^{1}\left(\widehat{X}, \mathcal{F}^{1}\right) \cong H^{1}\left(\widehat{X}, \mathcal{T}^{1}\left(X ; \mathcal{O}_{\widehat{X}}\right)\right)$. This can be seen directly as follows. We have the exact sequence

$$
\mathcal{T}^{0}\left(S ; \mathcal{O}_{\widehat{X}}\right) \rightarrow \mathcal{F}^{1} \rightarrow \mathcal{T}^{1}\left(X ; \mathcal{O}_{\widehat{X}}\right) \rightarrow 0
$$

As $S$ is smooth two-dimensional, the sheaf $\mathcal{T}^{0}\left(S ; \mathcal{O}_{\widehat{X}}\right)$ is isomorphic to $\mathcal{O}_{\widehat{X}} \oplus \mathcal{O}_{\widehat{X}}$ and therefore its $H^{1}$ vanishes, implying the isomorphism in question.

3.2 Let $C$ be the exceptional curve with its scheme structure defined, i.e., the one defined by $\mathfrak{m} \mathcal{O}_{\widehat{X}}$. We study the sheaves $\mathcal{F}^{i}$ with the exact sequence

$$
0 \rightarrow \mathfrak{m} \mathcal{F}^{i} \rightarrow \mathcal{F}^{i} \rightarrow \mathcal{F}_{\mid C}^{i} \rightarrow 0
$$

Proposition 3.2. For all $i$ one has

$$
\mathfrak{m} \mathcal{F}^{i} \cong \mathcal{T}^{i}\left(\widehat{X} / \widehat{S} ; \mathcal{O}_{\widehat{X}}\right) \otimes \mathcal{O}_{\widehat{X}}(i C)
$$

Proof. We describe $X$ with coordinates $\left(x, y ; z_{1}, \ldots, z_{m}\right)$, where $m=d-1$, and project onto the $(x, y)$-plane. The blow-up of the plane can be covered by two charts. By genericity of the projection we may assume that the two charts also suffice to cover $\widehat{X}$. One chart (which is a germ along the exceptional divisor, for which we take a Stein representative) has coordinates $\left(x, \eta ; t_{1}, \ldots, t_{m}\right)$ with $y=x \eta, z_{i}=x t_{i}$. On the second chart we have coordinates $\left(\xi, y ; t_{1}^{\prime}, \ldots, t_{m}^{\prime}\right)$ with $x=\xi y, z_{i}=y t_{i}^{\prime}$ so on the intersection $t_{i}=\xi t_{i}^{\prime}$.

We first look at the $x$-chart. We compute the stalk $\mathcal{F}_{y}^{i}$ from the Harrison complex $C_{\mathrm{sh}}\left(\mathcal{O}_{X, 0} / \mathcal{O}_{S, 0} ; \mathcal{O}_{\widehat{X}, y}\right)$. We write $C_{\mathrm{sh}}(A / P ; B)$ for short. An $(i+1)$-cochain $\varphi$ is determined by its values on $(i+1)$-tuples of coordinates $z_{j}$. For the sheaf $\mathcal{T}^{i}\left(\widehat{X} / \widehat{S} ; \mathcal{O}_{\widehat{X}}\right)$ we use the complex $C_{\mathrm{sh}}\left(\mathcal{O}_{\widehat{X}, y} / \mathcal{O}_{\widehat{S}, \tilde{\pi}(y)} ; \mathcal{O}_{\widehat{X}, y}\right)$ (for short we write $C_{\mathrm{sh}}^{\bullet}(B / Q ; B)$ ) with cochains determined by their values on $(i+1)$-tuples of coordinates $t_{j}$.

We define maps $s: C_{\mathrm{sh}}^{i+1}(A / P, B) \rightarrow C_{\mathrm{sh}}^{i+1}(B / Q, B)$ simply by

$$
(s \varphi)\left(t_{j_{0}}, \ldots, t_{j_{i}}\right)=\varphi\left(z_{j_{0}}, \ldots, z_{j_{i}}\right) .
$$

This does not give a map of complexes, but the following holds:

$$
s d=x d s,
$$




\section{Higher COTANGENT COHOMOLOGY}

where $x$ stands for the map 'multiplication by $x$ '. Let us check:

$$
\begin{aligned}
(s d \varphi)\left(t_{j_{0}}, \ldots, t_{j_{i}}\right) & =(d \varphi)\left(z_{j_{0}}, \ldots, z_{j_{i}}\right) \\
& =z_{j_{0}} \varphi\left(z_{j_{1}}, \ldots, z_{j_{i}}\right)-\varphi\left(z_{j_{0}} z_{j_{1}}, z_{j_{2}}, \ldots, z_{j_{i}}\right)+\ldots \\
& =z_{j_{0}} \varphi\left(z_{j_{1}}, \ldots, z_{j_{i}}\right)-\sum p_{j_{0} j_{1}}^{\nu}(x, y) \varphi\left(z_{\nu}, z_{j_{2}}, \ldots, z_{j_{i}}\right)+\cdots \\
& =x t_{j_{0}}(s \varphi)\left(t_{j_{1}}, \ldots, t_{j_{i}}\right)-\sum x \widehat{p}_{j_{0} j_{1}}^{\nu}(x, \eta)(s \varphi)\left(t_{\nu}, t_{j_{2}}, \ldots, t_{j_{i}}\right)+\cdots \\
& =x t_{j_{0}}(s \varphi)\left(t_{j_{1}}, \ldots, t_{j_{i}}\right)-x(s \varphi)\left(t_{j_{0}} t_{j_{1}}, t_{j_{2}}, \ldots, t_{j_{i}}\right)+\ldots \\
& =(x d s \varphi)\left(t_{j_{0}}, \ldots, t_{j_{i}}\right) .
\end{aligned}
$$

The maps $s$ induce a map $s_{*}$ on cohomology: if $d \varphi=0$, then $x d s \varphi=0$ and therefore $d s \varphi=0$ as $x$ is not a zero-divisor; furthermore, if $\varphi=d \psi$ then $s \varphi=d x s \psi$. As the maps $s$ are surjective, $s_{*}$ is also surjective.

The kernel of $s_{*}$ on $\operatorname{Harr}^{i+1}(A / P, B)$ is the kernel of multiplication by $x$ : if $s_{*}[\varphi]=0$, then $s \varphi=d s \psi$ for some $\psi$ and therefore $x \varphi=d \psi$; conversely if $x \varphi=d \psi$ then $x s \varphi=x d s \psi$, so $s_{*}[\varphi]=0$. This makes $\operatorname{Harr}^{i+1}(B / Q, B)$ isomorphic to the image $x \operatorname{Harr}^{i+1}(A / P, B)$, giving the claimed isomorphism locally.

To see what happens globally we also look at the $y$-chart, where we have the map $s^{\prime}$. If $\varphi \in \mathfrak{m} \mathcal{F}^{i}$ we write $\varphi\left(z_{j_{0}}, \ldots, z_{j_{i}}\right)=x \psi\left(z_{j_{0}}, \ldots, z_{j_{i}}\right)$ and the isomorphism maps it onto $(s \psi)\left(t_{j_{0}}, \ldots, t_{j_{i}}\right)=$ $\psi\left(z_{j_{0}}, \ldots, z_{j_{i}}\right)$. On the intersection we can write $\varphi\left(z_{j_{0}}, \ldots, z_{j_{i}}\right)=y \xi \psi\left(z_{j_{0}}, \ldots, z_{j_{i}}\right)$, so by the other isomorphism it gets mapped to a cocycle homologous to $\left(\xi s^{\prime} \psi\right)\left(t_{j_{0}}^{\prime}, \ldots, t_{j_{i}}^{\prime}\right)=\xi \psi\left(z_{j_{0}}, \ldots, z_{j_{i}}\right)$. By $\xi$-linearity we have that $(s \psi)\left(t_{j_{0}}^{\prime}, \ldots, t_{j_{i}}^{\prime}\right)=\xi^{i+1}(s \psi)\left(t_{j_{0}}, \ldots, t_{j_{i}}\right)=\xi^{i}\left(\xi s^{\prime} \psi\right)\left(t_{j_{0}}^{\prime}, \ldots, t_{j_{i}}^{\prime}\right)$.

Remark. If $i \geqslant 2$ then $\mathcal{T}^{i}\left(\widehat{X} / \widehat{S} ; \mathcal{O}_{\widehat{X}}\right) \cong \mathcal{T}_{\widehat{X}}^{i}$ is concentrated in points, but in general $\mathcal{T}^{1}\left(\widehat{X} / \widehat{S} ; \mathcal{O}_{\widehat{X}}\right)$ is not. Its support is the critical locus of the map $\widehat{X} \rightarrow \widehat{S}$. This follows from a local computation in smooth points of $\widehat{X}$ using the exact sequence

$$
\mathcal{T}_{\widehat{X}}^{0} \rightarrow \mathcal{T}^{0}\left(\widehat{S} ; \mathcal{O}_{\widehat{X}}\right) \rightarrow \mathcal{T}^{1}\left(\widehat{X} / \widehat{S} ; \mathcal{O}_{\widehat{X}}\right) \rightarrow \mathcal{T}_{\widehat{X}}^{1} \rightarrow 0 .
$$

In particular, if $C$ is reduced (i.e. $X$ has reduced fundamental cycle), then the support has no one-dimensional compact components and the $H^{1}$ of the sheaf vanishes.

Proposition 3.3. $H^{0}\left(\widehat{X}, \mathcal{F}_{\mid C}^{i}\right)=0$.

Proof. A global section of the sheaf $\mathcal{F}_{\mid C}^{i}$ consists of a collection of local sections $\varphi \in C_{\mathrm{sh}}^{i+1}(A / P, B)$ with $d \varphi=0$ such that the difference between two of them lies in the ideal of $C$. So each $\varphi\left(z_{j_{0}}, \ldots, z_{j_{i}}\right)$ gives rise to a global section of $\mathcal{O}_{C}$, which therefore is a constant. To prove the proposition we have to show that this constant is zero.

We now argue as in the proof of Lemma 1.5. In the $\operatorname{ring} B$ we have the equality

$$
\begin{aligned}
0= & (\delta f)\left(z_{j_{0}}, \ldots, z_{j_{i}}, z_{k}\right) z_{j_{0}} \varphi\left(z_{j_{1}}, \ldots, z_{j_{i}}\right) \pm z_{k} f\left(z_{j_{0}}, \ldots, z_{j_{i}}\right) \\
& -\sum p_{j_{0} j_{1}}^{\nu}(x, y) \varphi\left(z_{\nu}, z_{j_{2}}, \ldots, z_{j_{i}}\right)+\cdots
\end{aligned}
$$

and we may divide by $x$ to obtain

$$
0=t_{j_{0}} \varphi\left(z_{j_{1}}, \ldots, z_{j_{i}}\right) \pm t_{k} f\left(z_{j_{0}}, \ldots, z_{j_{i}}\right)-\sum \widehat{p}_{j_{0} j_{1}}^{\nu}(x, \eta) \varphi\left(z_{\nu}, z_{j_{2}}, \ldots, z_{j_{i}}\right)+\cdots
$$

We note that $C$ is a principal divisor, defined by $x$. As $1, t_{1}, \ldots, t_{d-1}$ are linearly independent modulo $(x, \eta)$, we find that $\varphi\left(z_{j_{1}}, \ldots, z_{j_{i}}\right)=0 \in H^{0}\left(\mathcal{O}_{C}\right)$.

Corollary 3.4. For $i \geqslant 2$ one has $H^{0}\left(\mathcal{F}^{i}\right) \cong H^{0}\left(\mathcal{T}_{\widehat{X}}^{i}\right)$. 


\section{J. Stevens}

3.3 In order to compute $H^{1}\left(\mathcal{F}^{i-1}\right)$ we identify this group with the kernel of the map $T^{i}\left(X / S ; \mathcal{O}_{X}\right) \rightarrow$ $H^{0}\left(\mathcal{F}^{i}\right)$. From now on we fix an element $x$, which is supposed to be chosen generically.

Lemma 3.5. The kernel of multiplication by $x$ on $T^{i}\left(X / S ; \mathcal{O}_{X}\right)$ is contained in the kernel of the $\operatorname{map} T^{i}\left(X / S ; \mathcal{O}_{X}\right) \rightarrow H^{0}\left(\mathcal{F}^{i}\right)$.

Proof. Suppose $\varphi \in T^{i}\left(X / S ; \mathcal{O}_{X}\right)$ is annihilated by $x$, i.e. $x \varphi=d \psi$. As $d \psi \equiv 0$ modulo $P$, the argument of Lemma 1.5 shows that $\psi$ takes values in the maximal ideal.

Consider $\varphi$ as a global section of $\mathcal{F}^{i}$. We may assume by genericity of $x$ that a global section vanishes if and only if it vanishes in the chart $x \neq 0$ (the special points of $\mathcal{F}^{i}$ on the exceptional divisor lie in this chart). The values of $\psi$ lie in $\mathfrak{m}$ and are therefore divisible by $x$ in $B$. We obtain that $\varphi=d(\psi / x)$.

Lemma 3.6. Let $K$ be a submodule of $T_{X}^{i}, i \geqslant 2$, containing the kernel of multiplication by $x$. Then $\operatorname{dim} K / x K=\operatorname{cg} T^{i}$.

Proof. Consider the multiplication $K \stackrel{\cdot x}{\rightarrow} K$. As $K \subset T^{i}$ the kernel is always contained in $\operatorname{Ker}\left\{T^{i} \stackrel{\cdot x}{\rightarrow}\right.$ $T^{i}$, so under the assumption of the lemma both kernels are equal. As $K$ is finite-dimensional, kernel and cokernel have the same dimension, and the same holds for $T^{i}$. Therefore, $\operatorname{dim} K / x K=$ $\operatorname{dim} T^{i} / x T^{i}$. For a general hyperplane section this dimension is $\operatorname{cg} T^{i}$.

The previous two lemmas show that $\operatorname{dim} H^{1}\left(\mathcal{F}^{i-1}\right) / x H^{1}\left(\mathcal{F}^{i-1}\right)$ has dimension $f_{i}(d)$. We have to determine $x H^{1}\left(\mathcal{F}^{i-1}\right)$. Since $x$ is generic the cokernel of $\mathcal{F}^{i-1} \stackrel{\cdot x}{\rightarrow} \mathfrak{m} \mathcal{F}^{i-1}$ has support at the strict transform of the divisor of $x$. In particular, its $H^{1}$ vanishes and therefore the map $H^{1}\left(\mathcal{F}^{i-1}\right) \stackrel{\cdot x}{\rightarrow}$ $H^{1}\left(\mathfrak{m} \mathcal{F}^{i-1}\right)$ is surjective. The image of the composed map $H^{1}\left(\mathcal{F}^{i-1}\right) \stackrel{\cdot x}{\rightarrow} H^{1}\left(\mathfrak{m} \mathcal{F}^{i-1}\right) \rightarrow H^{1}\left(\mathcal{F}^{i-1}\right)$ is $x H^{1}\left(\mathcal{F}^{i-1}\right)$. As $H^{1}\left(\mathfrak{m} \mathcal{F}^{i-1}\right) \rightarrow H^{1}\left(\mathcal{F}^{i-1}\right)$ is injective we obtain that $x H^{1}\left(\mathcal{F}^{i-1}\right)=H^{1}\left(\mathfrak{m} \mathcal{F}^{i-1}\right)$. For $i>2$ this group vanishes.

3.4 To collect our results into formulas for the dimension of $T^{i}$ we introduce a name for the remaining unknown term.

Definition 3.7. For a rational surface singularity $X$ we define the invariant

$$
c(X)=\operatorname{dim} H^{1}\left(\mathfrak{m} \mathcal{F}^{1}\right) .
$$

Remarks.

(1) If $X$ has reduced fundamental cycle, the correction term $H^{1}\left(\mathfrak{m} \mathcal{F}^{1}\right)=H^{1}\left(\widehat{X}, \mathcal{T}^{1}\left(\widehat{X} / \widehat{S} ; \mathcal{O}_{\widehat{X}}\right)(C)\right)$ vanishes by the remark in $\S 3.2$.

(2) Our invariant is the same as the one Christophersen and Gustavsen [CG01] define using their absolute version $\mathcal{F}_{C G}^{1}$ of the sheaf $\mathcal{F}^{1}$. This follows from the formula for $T^{2}$. A direct proof can be obtained as in the remark in $\S 3.1$. One tensors the exact sequence occurring there with the invertible sheaf $\mathcal{O}_{\widehat{X}}(-C)$ and uses the fact that $H^{1}\left(\widehat{X}, \mathfrak{m} \mathcal{F}_{C G}^{1}\right)=H^{1}\left(\widehat{X}, \mathcal{F}_{C G}^{1}(-C)\right)[\mathrm{CG} 01$, Proposition 4.1].

THeOREM 3.8. If $X$ is a rational surface singularity of multiplicity $d$ and $\widehat{X} \rightarrow X$ the first blow-up, then for $i>2$

$$
\operatorname{dim} T_{X}^{i}=\operatorname{cg} T_{X}^{i}+\operatorname{dim} T_{\widehat{X}}^{i}
$$

with $\operatorname{cg} T_{X}^{i}=f_{i}(d)$ as given in $\S 1.6$, and

$$
\operatorname{dim} T_{X}^{2}=(d-1)(d-3)+\operatorname{dim} T_{\widehat{X}}^{2}+c(X) .
$$




\section{Higher COTANGENT COHOMOLOGY}

\section{Good maximal deformations}

4.1 The dimension formula above is an inductive formula. To make it more explicit we first define the multiplicity sequence of a rational singularity in the obvious way as the sequence of multiplicities of the singularities on successive blow-ups (this are the infinitely near singularities, including the singularity itself). We denote by $X_{P}$ the singularity at an infinitely near point $P$ and by $d(P)$ its multiplicity.

The $T^{1}$-formula of [CG01] can be best stated as a formula for the codimension of the (smooth) Artin component in the Zariski tangent space of the versal base space. We denote this invariant by $\operatorname{cod}_{A C}(X)$. For the cone over the rational normal curve of degree $d \geqslant 3$ it has value $d-3$.

By induction we obtain the following formulas from [CG01, Theorems 3.8 and 3.13] with Theorem 3.8 .

Theorem 4.1. For a rational surface singularity $X$ of multiplicity $d$

$$
\begin{aligned}
\operatorname{dim} T_{X}^{i} & =\sum_{P} f_{i}(d), \quad i \geqslant 3, \\
\operatorname{dim} T_{X}^{2} & =\sum_{P}(d(P)-1)(d(P)-3)+\sum_{P} c\left(X_{P}\right), \\
\operatorname{cod}_{A C}(X) & =\sum_{P}(d(P)-3)+\sum_{P} c\left(X_{P}\right),
\end{aligned}
$$

where the sum ranges over all infinitely near singular points $P$ of multiplicity at least three.

4.2 De Jong and Van Straten [JS94] derived their dimension formulas for $T^{1}$ and $T^{2}$ using a special deformation (on the Artin component) to the cone over the rational normal curve of degree $d$ and all singularities on the first blow-up. The same deformation also yields the surjectivity of the obstruction map. For any deformation $X_{S} \rightarrow S$ of an isolated singularity $X$ one has an obstruction map $\left(\mathcal{J} / \mathfrak{m}_{S} \mathcal{J}\right)^{*} \rightarrow T_{X}^{2}$ [Sch73], see also [JS94, § 4], where $\mathcal{J}$ is the ideal defining $S$ in its minimal embedding. To define it we use the definition of $T^{2}$ in terms of functions on relations. Let $\sum F_{i} R_{i j} \equiv 0(\bmod \mathcal{J})$ be a lift of the relation $\sum f_{i} r_{i j}=0$ between the generators of the ideal of $X$. Let $\left\{g_{k}\right\}_{k \in K}$ be the generators of $\mathcal{J}$ and $l: \mathcal{J} / \mathfrak{m}_{S} \mathcal{J} \rightarrow \mathbb{C}$ be a linear form. Then $\sum F_{i} R_{i j}=\sum a_{m k} g_{k}$ and we get a linear function on relations by sending the $j$ th relation $\sum f_{i} r_{i j}=0$ to $\sum \bar{a}_{m k} l\left(g_{k}\right)$, where $\bar{a}_{m k}$ is the reduction modulo $\mathfrak{m}_{S}$. As an element of $T^{2}$ this function is well defined. Suppose now that the first-order infinitesimal deformation over $T_{0} S$ is isomorphic to the universal firstorder family over $T_{X}^{1}$, then the deformation $X_{S} \rightarrow S$ is versal if the obstruction map is injective. Surjectivity of the obstruction map means then that the number of generators of $\mathcal{J}$ equals the dimension of $T_{X}^{2}$.

For general singularities the obstruction map is not surjective; a specific example is the fat point $Z_{3}$, see $[\mathrm{BC} 91, \S 6.2 .2]$. For rational surface singularities no example is known where the obstruction map is not surjective. The only proofs are based on direct calculation or the use of special deformations [JS94, Jon98]. The important ingredient in our dimension formulas is the multiplicity sequence. With this in mind we define a more general class of special deformations.

Definition 4.2. A good maximal deformation of a rational surface singularity $X$ of multiplicity $d$ is a one-parameter deformation $X_{T} \rightarrow T$ such that the general fibre $X_{t}$ has as singularities cones over 


\section{J. Stevens}

rational normal curves of multiplicity $d(P)$, one for each infinitely near singularity of multiplicity at least three.

Proposition 4.3. If a rational surface singularity $X$ has a good maximal deformation, then the number of equations of the versal base space equals the dimension of $T_{X}^{2}$.

Proof. Let $X_{T} \rightarrow T$ be a good maximal deformation of $X$. Versality induces a map $b: T \rightarrow B_{X}$, to the base space of the miniversal deformation. The embedding dimension of $B_{X}$ is $\operatorname{dim} T_{X}^{1}=\tau$, and $B_{X}$ can be realized as a fibre of a nonlinear map $T_{X}^{1} \rightarrow T_{X}^{2}$. In a general point $b(t)$ the embedding dimension of the germ $\left(B_{X}, b(t)\right)$ is $\tau-\sum_{P} c\left(X_{P}\right)$. The number of generators of the ideal of $\left(B_{X}, b(t)\right)$ in its minimal embedding is $\sum_{P}(d(P)-1)(d(P)-3)$, and one needs $\sum_{P} c\left(X_{P}\right)$ equations with independent linear parts to describe the space in which $\left(B_{X}, b(t)\right)$ is embedded. Therefore one needs at the origin at least $\sum_{P}(d(P)-1)(d(P)-3)+\sum_{P} c\left(X_{P}\right)$ equations. As this number is the dimension of $T_{X}^{2}$, they also suffice.

Remark. The $T^{1}$-formula above follows easily from the existence of a good maximal deformation. Consider the long exact sequence

$$
T_{X_{T} / T}^{1} \stackrel{\alpha}{\rightarrow} T_{X}^{1} \rightarrow T_{X_{T} / T}^{2} \stackrel{\cdot t}{\rightarrow} T_{X_{T} / T}^{2} \stackrel{\beta}{\rightarrow} T_{X}^{2} \rightarrow T_{X_{T} / T}^{3} \stackrel{\cdot t}{\rightarrow} T_{X_{T} / T}^{3} \stackrel{\gamma}{\rightarrow} T_{X}^{3} .
$$

The dimension of $T^{3}$ is constant in this deformation, so the rank of the $\mathbb{C}\{t\}$-module $T_{X_{T} / T}^{3}$ is equal to $\operatorname{dim} T_{X}^{3}$. Therefore, $\gamma$ is surjective and, as the rank is equal to $\operatorname{dim} \operatorname{Coker}(\cdot t)-\operatorname{dim} \operatorname{Ker}(\cdot t)$, we obtain that multiplication by $t$ is injective, and therefore the map $\beta$ is also surjective. By assumption on the deformation we have $\operatorname{dim} T_{X}^{2}-\operatorname{Rank} T_{X_{T} / T}^{2}=\sum_{P} c\left(X_{P}\right)$. The dimension of $\operatorname{Ker}\left(\cdot t: T_{X_{T} / T}^{2} \rightarrow\right.$ $\left.T_{X_{T} / T}^{2}\right)$ therefore also equals $\sum_{P} c\left(X_{P}\right)$ and we obtain $\operatorname{dim} T_{X}^{1}=\operatorname{dim} \operatorname{Im} \alpha+\sum_{P} c\left(X_{P}\right)$. But we know the dimension of the image of $\alpha$ : by [GL85] it equals the dimension of the Zariski tangent space to $B_{X}$ in the general point $b(t)$. The codimension of the Artin component is therefore $\sum_{P}(d(P)-3)$.

4.3 The existence of a good maximal deformation has been established by De Jong and Van Straten for rational singularities with reduced fundamental cycle and by De Jong in the determinantal case [Jon98].

In the first case one can deform to the singularities on the first blow-up plus a cone of degree $d$, but for determinantal singularities this is in general impossible. Specifically, if $X$ contains one configuration of type $D_{2 d+1}^{\mathrm{II}}$ (in the notation of [Jon98]), then the dimension of $T^{1}$ will be too small to allow a deformation to a determinantal with $A_{2 d^{-}}^{1}$ configuration and a cone (this was checked by computing $T^{1}$ in the case $d=3$ ). However, the singularity does deform into two cones.

In the definition of a good maximal deformation we ignore all occurring rational double points, for a good reason: the singularities on the first blow-up of $D_{4}$ are three $A_{1}$ 's, and the cone over the rational normal curve of degree two is also $A_{1}$ but there is no deformation $D_{4} \rightarrow 4 A_{1}$.

It was hoped that every rational singularity has a good maximal deformation but unfortunately this is not true. First we note that the dimension of the Artin component equals $h^{1}\left(\widetilde{X}, \Theta_{\tilde{X}}\right)$, where $\widetilde{X} \rightarrow X$ is the minimal resolution, with exceptional set $E=E_{1} \cup \cdots \cup E_{r}$. As usual we denote by $-b_{i}$ the self-intersection of the irreducible component $E_{i}$. By [Wah76, Propositions 2.2 and 2.5],

$$
h^{1}\left(\widetilde{X}, \Theta_{\widetilde{X}}\right)=\sum_{i=1}^{r}\left(b_{i}-1\right)+h^{1}\left(\widetilde{X}, \Theta_{\widetilde{X}}(\log E)\right) .
$$

The second summand gives the dimension of the equisingular stratum. The stratum in the Artin component of fibres with a cone over the rational normal curve of multiplicity $d$ as singularity has codimension $d-1$. By openness of versality these strata intersect transversally in the base of a good maximal deformation. 


\section{Higher COTANGENT COHOMOLOGY}

Lemma 4.4. The general rational singularity with a given resolution graph does not have a good maximal deformation if $\sum_{P}(d(P)-1) \geqslant \sum\left(b_{i}-1\right)$.

An example where this condition is satisfied, is obtained by generalizing the $D_{4}$-singularity to higher multiplicity: consider a singularity with fundamental cycle reduced everywhere except at one $(-2)$-curve, such that the first blow-up has three singularities of multiplicity $k$. The simplest way to do this gives the following graph, where as usual a dot stands for a (-2)-curve:

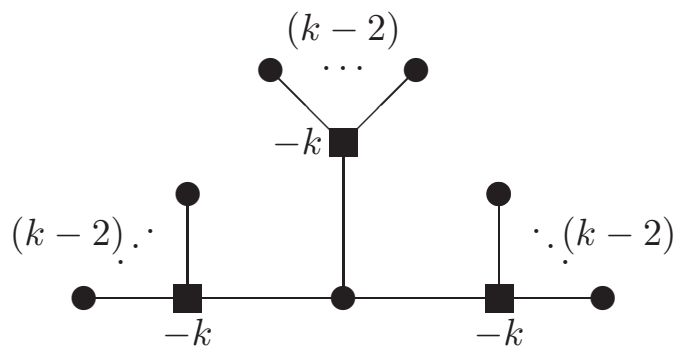

The singularity has multiplicity $3 k-4$; for $k=2$ it is indeed $D_{4}$. Here we have $\sum_{P}(d(P)-1)=$ $\sum\left(b_{i}-1\right)=6 k-8$.

\section{ACKNOWLEDGEMENTS}

I am grateful to Jan Christophersen for discussions and comments.

\section{REFERENCES}

AS99 Klaus Altmann and Jan Stevens, Cotangent cohomology of rational surface singularities, Invent. Math. 138 (1999), 163-181.

And74 Michel André, Homologie des algèbres commutatives, Grundlehren der mathematischen Wissenschaften, vol. 206 (Springer, Berlin, 1974).

BC91 Kurt Behnke and Jan Arthur Christophersen, Hypersurface sections and obstructions (rational surface singularities), Compositio Math. 77 (1991), 233-268.

CG01 Jan A. Christophersen and Trond S. Gustavsen, On infinitesimal deformations and obstructions for rational surface singularities, J. Algebraic Geom. 10 (2001), 179-198.

GL85 G.-M. Greuel and E. Looijenga, The dimension of smoothing components, Duke Math. J. 52 (1985), 263-272.

Jon98 Theo de Jong, Determinantal rational surface singularities, Compositio Math. 113 (1998), 67-90.

JS94 Theo de Jong and Duco van Straten, On the deformation theory of rational surface singularities with reduced fundamental cycle, J. Algebraic Geom. 3 (1994), 117-172.

Lod92 Jean-Louis Loday, Cyclic Homology, Grundlehren der mathematischen Wissenschaften, vol. 301 (Springer, Berlin, 1992).

Pal76 V. P. Palamodov, Deformations of complex spaces, Russ. Math. Surveys 31 (1976), 129-197 (translation from Usp. Mat. Nauk 31 (1976), 129-194).

Pal84 V. P. Palamodov, The tangent complex of an analytic space, Amer. Math. Soc. Transl. 122 (1984), 119-171 (translation from Trudy Sem. Petrovsk. 4 (1978), 173-226).

Pal83 V. P. Palamodov, Cohomology of analytic algebras, Trans. Mosc. Math. Soc. 2 (1983), 1-61 (translation from Tr. Mosk. Mat. O.-va 44 (1982), 3-61).

Pal90 V. P. Palamodov, Deformations of complex spaces, in Several complex variables. IV. Algebraic aspects of complex analysis, Encycl. Math. Sci. 10 (Springer, Berlin, 1990), 105-194 (translation from Itogi Nauki Tekh., Ser. Sovrem. Probl. Mat., Fundam. Napravleniya 10 (1986), 123-221). 


\section{Higher COTANGENT COHOMOLOGY}

Sch73 Michael Schlessinger, On rigid singularities, Rice Univ. Stud. 59 (1973), 147-162.

Wah76 Jonathan M. Wahl, Equisingular deformations of normal surface singularities, I, Ann. Math. 104 (1976), 325-356.

Jan Stevens stevens@math.chalmers.se

Matematik, Göteborgs universitet, Chalmers tekniska högskola, SE-412 96 Göteborg, Sweden 